\title{
The past, present, and future of 'omics analyses in ocean biogeochemistry.
}

$$
\text { BETHANIE R EDWARDS }
$$

\section{UC-Berkeley}

Presenting Author: bethanie_edwards@berkeley.edu

The integrated -omics revolution that is underway in aquatic microbial ecology was catalyzed by advances in next generation sequencing, high-resolution accurate-mass (HRAM) mass spectrometry, and our capacity to work with big datasets in statistically relevant ways. Here we will begin with a practical overview of analyzing sequence based -omes, like genomes and transcriptomes, and mass-spec based -omes, like proteomes and metabolomes (both water soluble metabolites and lipids), diving into the challenges present by both -omic types. When applied in the environment, integrated meta-omic approaches allow us to move through the central dogma of molecular biology (DNA -> RNA $->$ protein) towards an understanding of microbial metabolism and biogeochemical fluxes, leading to observations that can be understood at a mechanic level through model system studies in the lab or mesocosms. To gain a historical appreciation, we will walk through a case study of -omic analyses employed in aquatic ecosystems from early studies of lipid that fringe on meta-lipidomics to modern integrated multi omic studies at time-series sites, across environmental gradients, and during Lagrangian process studies. Lastly, we will look to the future, highlighting recent advances in methodology and opportunities to accelerate the field by employing machine learning, autonomous sampling platforms, leveraging advances in biomedical applications of -omics, and building databases focused on aquatic and marine analytes. 